\title{
Risk and Protective Factors for Cholera Deaths during an Urban Outbreak—Lusaka, Zambia, 2017-2018
}

\author{
Lwito Salifya Mutale, ${ }^{1} \dagger$ Alison V. Winstead, ${ }^{2 \star} \dagger$ Patrick Sakubita, ${ }^{1}$ Fred Kapaya, ${ }^{1}$ Sulani Nyimbili, ${ }^{3}$ Nelia L. Mulambya, ${ }^{1}$ \\ Francis H. Nanzaluka, ${ }_{1}$ Angela Gama, ${ }^{1}$ Vivian Mwale, ${ }^{1}$ Sunkyung Kim, ${ }^{2}$ William Ngosa, ${ }^{4}$ Ellen Yard, ${ }^{1,5}$ Nyambe Sinyange, ${ }^{1,4}$ \\ Eric Mintz, ${ }^{2}$ Joan Brunkard, ${ }^{2} \ddagger$ and Victor Mukonka ${ }^{4} \ddagger$ \\ ${ }^{1}$ Zambia Field Epidemiology Training Program, Lusaka, Zambia; ${ }^{2}$ Centers forDisease Control and Prevention, Atlanta, Georgia; ${ }^{3}$ Ministry of Health, \\ Lusaka, Zambia; ${ }^{4}$ Zambia National Public Health Institute, Lusaka, Zambia; ${ }^{5}$ Centers for Disease Control and Prevention, Lusaka, Zambia
}

\begin{abstract}
The Republic of Zambia declared a cholera outbreak in Lusaka, the capital, on October 6, 2017. By midDecember, 20 of 661 reported cases had died (case fatality rate $3 \%$ ), prompting the CDC and the Zambian Ministry of Health through the Zambia National Public Health Institute to investigate risk factors for cholera mortality. We conducted a study of cases (cholera deaths from October 2017 to January 2018) matched by age-group and onset date to controls (persons admitted to a cholera treatment center [CTC] and discharged alive). A questionnaire was administered to each survivor (or relative) and to a family member of each decedent. We used univariable exact conditional logistic regression to calculate matched odds ratios (mORs) and 95\% Cls. In the analysis, 38 decedents and 76 survivors were included. Median ages for decedents and survivors were 38 (range: 0.5-95) and 25 (range: 1-82) years, respectively. Patients aged $>55$ years and those who did not complete primary school had higher odds of being decedents (matched odds ratio [mOR] 6.3, 95\% Cl: 1.2-63.0, $P=0.03$; mOR 8.6, 95\% Cl: 1.8-81.7, $P<0.01$, respectively). Patients who received immediate oral rehydration solution (ORS) at the CTC had lower odds of dying than those who did not receive immediate ORS (mOR 0.1, 95\% Cl: 0.0-0.6, $P=0.02$ ). Cholera prevention and outbreak response should include efforts focused on ensuring access to timely, appropriate care for older adults and less educated populations at home and in health facilities.
\end{abstract}

\section{INTRODUCTION}

Cholera is endemic in more than 40 countries globally, with an estimated 1.3-4.0 million cholera cases and 21,000-143,000 cholera deaths each year, most of which occur in developing countries. ${ }^{1}$ The first cholera outbreak recorded in Zambia occurred in 1977 with 178 cases; cholera has been recorded nearly every year since then. ${ }^{2}$ Zambia experienced outbreaks of more than 10,000 cases in 1991, 1992, 1999, and 2004. ${ }^{3}$ Environmental conditions conducive to cholera spread, such as lack of access to safe water and improved sanitation, are common in Zambia, particularly in remote regions and in high-density, periurban neighborhoods of Lusaka, the capital city. ${ }^{4,5}$

Cholera is caused by consuming food or water contaminated by toxigenic Vibrio cholerae serogroup 01 or 0139. Most people exposed to these pathogens do not develop symptoms. However, approximately $25 \%$ will experience the acute onset of watery diarrhea, ${ }^{6}$ which in about one-third of symptomatic cases will be profuse and accompanied by vomiting. Without treatment, severe dehydration will ensue with case fatality rates (CFRs) that can reach up to $50 \%$. With appropriate oral and intravenous rehydration, the CFR can be less than $1 \%{ }^{7}$

A cholera outbreak, centered predominantly in Lusaka, was declared on October 6, 2017. Lusaka district has an estimated population of 1.7 million, with a population density of 4,853 people per square kilometer as of $2010 .^{8}$ Within Lusaka Province, it had been estimated that $24 \%$ live in poverty and $66 \%$ lack basic and safely managed sanitation. ${ }^{8,9}$ Although cases were eventually recorded in seven of 10 provinces in Zambia, most cases outside Lusaka had epidemiological links with

${ }^{*}$ Address correspondence to Alison V. Winstead, Centers for Disease Control and Prevention, 1600 Clifton Rd., Mailstop E92, Atlanta, GA 30333. E-mail: nrc0@cdc.gov

†These authors contributed equally to this manuscript.

$\ddagger$ These senior authors contributed equally to this manuscript. cases in Lusaka. By December 11, 20 of 661 reported cases had died (CFR 3\%), prompting the CDC and the Zambian Ministry of Health (MoH) through the Zambia National Public Health Institute to conduct a case-control study to identify factors associated with mortality and determine the interventions that could best prevent future cholera-related deaths.

\section{MATERIALS AND METHODS}

We conducted a retrospective case-control study from January to March 2018. Decedents were patients who died from cholera (defined as severe dehydration or death from acute watery diarrhea, regardless of age) in Lusaka district from October 1, 2017 to January 15, 2018. The deaths occurred in either a cholera treatment center (CTC) or the community. A community decedent was defined as a person who died in the community or who died with suspected cholera within 45 minutes of arrival at a CTC. Survivors were cholera patients admitted to a CTC in Lusaka district during the same period who were discharged alive. Decedents and survivors were identified through a convenience sampling of those included on the $\mathrm{MoH}$ cholera line list. In addition, some decedents were identified before their inclusion on the line list through multiple sources, including review of patient records at CTCs and information from community health workers. We attempted to include all cholera deaths occurring during the specified time period. We assigned two survivors to each decedent, matched by age-group ( $<2,2-17, \geq 18$ years) and date of onset $( \pm 1$ day). If more than two survivors met the matching criteria, two survivors were randomly selected from eligible controls. If fewer than two survivors met the matching criteria, the matching criterion for date of onset was extended to \pm 5 days. We excluded decedents and survivors if they did not reside in Lusaka district, if the patient or family member did not consent for interview, if the patient originally designated as deceased was found alive, or if the patient or a family member was unable to be located. 
Interviews were conducted in the home of the patient or respondent. For survivors who were unavailable for interview and decedents, we attempted to interview close family members who had knowledge of the patient's history and clinical course. We obtained written consent from all participants aged $\geq 18$ years before the interview. Parents or guardians of participants aged $<18$ years were asked for consent, and assent was obtained from all adolescents aged 13-17 years. Questionnaires were written in English and distributed electronically to tablets used by enumerators. Questions were translated into standardized Nyanja or Bemba by enumerators during the interview to ensure understanding.

Information collected included demographics, education level, socioeconomic indicators, comorbidities, symptoms and clinical course of illness, care-seeking behaviors, cholera treatment at home and at any health facilities, knowledge about cholera, cholera prevention activities, and water, sanitation, and hygiene characteristics and behaviors at the household.

In addition to information collected during the case-control study, we reviewed available medical records of cholera decedents from the CTCs, abstracting data on illness onset, symptoms, length of stay, comorbidities, and treatment received. Medical records of all decedents were requested from each CTC from the beginning of the outbreak until the time of data collection (January 9-26, 2018).

The Zambian MoH Research Ethics Committee approved the study protocol, and the project was reviewed in accordance with CDC human research protection procedures and was determined to be a non-research outbreak response activity.

Statistical analysis. Data for the case-control study were collected using Epi Info 7 (CDC, Atlanta, GA), cleaned using STATA, version 13.0 SE (Stata Corporation LP, College Station, TX), and analyzed using SAS v9.4 (Cary, NC). To explore factors associated with cholera mortality, we used exact conditional logistic regression considering the matched case-control study design and small sample size. In each univariable analysis, survivorship status (deceased or alive) was treated as a dependent variable and a factor was treated as an independent variable. Matched odds ratios and $95 \% \mathrm{Cls}$ were obtained. Exclusion of community decedents $(n=17)$ was deemed logical for some analyses (e.g., duration of hospital stay) and is noted where applied in the results. Analyses of clinical and treatment data were restricted to decedents who received treatment at a CTC and to their matched survivors. Age analysis was conducted only on age $\geq 18$ years, as age was not matched in that age-group, and the only matching variable for that analysis was date of onset. Only adults aged $\geq 18$ years were considered for analysis of education level. Employment status was only analyzed for those aged 16-65 years. For continuous variables, the Wilcoxon rank-sum test was applied. For the medical record review, we abstracted records using Open Data Kit (ODK, University of Washington, Seattle, WA, https://opendatakit.org/).

\section{RESULTS}

During this 6-month-long cholera outbreak, 5,905 suspected cases and 114 deaths were reported. Most cases $(5,414$ [91.7\%]) and deaths (98 [86.0\%]) were reported by Lusaka district (Figure 1). A total of 38 decedents and 76 survivors (matched 1:2) were included in the study. For the decedents, proxies interviewed were parents (34\%), spouses (18\%), children (13\%), siblings (11\%), in-laws (11\%), aunts or uncles $(8 \%)$, and grandmothers (5\%). Most (62\%) survivor respondents were also proxies. Proxies interviewed for survivors were parents $(29 \%)$, spouses $(8 \%)$, children $(7 \%)$, siblings $(8 \%)$, other friend or family member $(4 \%)$, in-laws $(3 \%)$, aunts or uncles $(2 \%)$, and grandmothers (1\%).

Demographics. When analyzing all participants included in the study, decedents were older than survivors (median ages: 38 and 25 years, respectively). With analysis restricted to age $\geq 18$ years, a higher proportion of decedents were aged more than 55 years as compared with survivors (26\% versus $4 \%)$ and a higher proportion of decedents did not complete primary education (54\% versus 16\%). Most participants used a pit latrine that was shared with other households (95\% for decedents and $81 \%$ for survivors) and had not been previously vaccinated against cholera $(87 \%$ for decedents and $86 \%$ for survivors). In matched analyses, patients aged $>55$ years and patients who did not complete primary school had higher odds of being decedents (mOR 6.3, 95\% Cl: 1.2-63.0, $P=0.03$; mOR $8.6,95 \% \mathrm{Cl}: 1.8-81.7, P<0.01$, respectively) (Table 1). There was no statistical association between cholera death and gender, employment status, household size, household assets, primary water source, type of toilet, or prior cholera vaccination status. Patients with household ownership of a smart phone had lower odds of being decedents (mOR 0.4, 95\% Cl: 0.1-1.0, $P=0.06$ ).

Clinical presentation and treatment. Of the 38 decedents, 21 (55\%) died in CTCs and 17 (45\%) died in the community. Similar proportions of decedents (40\%) and survivors (36\%) received ORS at home before presentation to a health facility, and a similar average time from onset of symptoms of initiation of ORS at home was seen for decedents (median: 2 hours [IQR 1-7]) and survivors (median: 2 hours [IQR 1-5]) (Table 2). Similar proportions of decedents $(21 \%)$ and survivors (17\%) received an antibiotic at home, but most of these patients $(89 \%)$ reported taking metronidazole, which is not recommended for the treatment of cholera. Two patients (11\%) took co-trimoxazole, a recommended antibiotic, at home, but later died from their illnesses. Through interviews, we could discern no differences between the clinical presentations of decedents and survivors that reached statistical significance. The majority experienced diarrhea and vomiting, and less than half

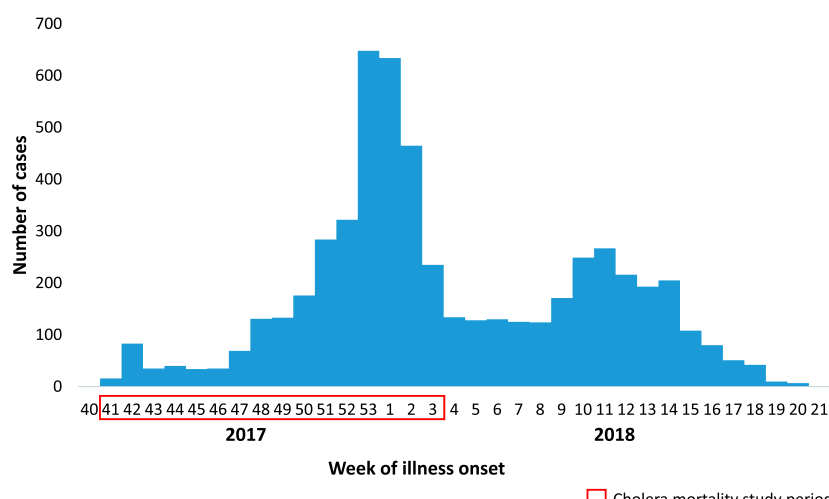

FIGURE 1. Epidemiologic curve showing suspected cases of cholera during the outbreak by week of illness onset-Lusaka district, Zambia, 2017-2018. This figure appears in color at www.ajtmh.org. 
TABLE 1

Demographic characteristics of cholera patients, by survivorship status-Lusaka, Zambia, 2017-2018

\begin{tabular}{|c|c|c|c|c|}
\hline & \multirow{2}{*}{$\frac{\text { Decedents }(n=38)}{n^{\star}(\%)}$} & \multirow{2}{*}{$\frac{\text { Survivors }(n=76)}{n^{\star}(\%)}$} & \multirow[b]{2}{*}{ Matched odds ratio $(95 \% \mathrm{Cl})$} & \multirow[b]{2}{*}{$P$-value } \\
\hline & & & & \\
\hline Age (years), median [IQR] & $38[7-57]$ & 25 [12-32] & - & - \\
\hline \multicolumn{5}{|l|}{ Older age (years) } \\
\hline$>55$ & $10(26)$ & $3(4)$ & $6.3(1.2-63.0) \dagger$ & 0.03 \\
\hline$\leq 55$ & $28(74)$ & $73(96)$ & Ref & - \\
\hline \multicolumn{5}{|l|}{ Gender } \\
\hline Male & $21(53)$ & $43(57)$ & $0.9(0.4-2.2)$ & 1.00 \\
\hline Female & $17(47)$ & $33(43)$ & Ref & \\
\hline \multicolumn{5}{|l|}{ Education $\ddagger$} \\
\hline$<$ Primary & $12(54)$ & $8(16)$ & $8.6(1.8-81.7)$ & $<0.01$ \\
\hline$\geq$ Primary & $14(46)$ & $43(84)$ & Ref & - \\
\hline \multicolumn{5}{|l|}{ Employment status§ } \\
\hline Employed & $13(59)$ & $33(67)$ & $0.6(0.2-2.3)$ & 0.62 \\
\hline Not employed & $9(41)$ & $16(33)$ & Ref & - \\
\hline Household size (persons), median [IQR] & $5[4-6]$ & $5[3-6]$ & - & - \\
\hline$\geq 6$ & $16(44)$ & $31(41)$ & $1.1(0.5-2.7)$ & 0.94 \\
\hline$<6$ & $20(56)$ & $44(59)$ & Ref & - \\
\hline \multicolumn{5}{|l|}{ Household assets\| } \\
\hline Bank account & $10(27)$ & $14(19)$ & $1.5(0.5-4.1)$ & 0.56 \\
\hline Smartphone & $6(16)$ & 27 (36) & $0.4(0.1-1.0)$ & 0.06 \\
\hline Animals & $5(14)$ & $5(7)$ & $2.2(0.5-11.3)$ & 0.40 \\
\hline Bicycle & $5(14)$ & $9(12)$ & $1.3(0.3-4.3)$ & 0.90 \\
\hline Car & $0(0)$ & $5(7)$ & $0.3(0-1.6)$ & 0.26 \\
\hline Laptop & $0(0)$ & $6(8)$ & $0.2(0-1.3)$ & 0.18 \\
\hline \multicolumn{5}{|l|}{ Primary water source } \\
\hline Kiosk & $25(68)$ & $38(50)$ & $2.1(0.8-5.4)$ & 0.12 \\
\hline Piped water & 7 (19) & $15(20)$ & $1.0(0.3-3.2)$ & 1.00 \\
\hline Borehole & $4(11)$ & $19(25)$ & $0.4(0.1-1.2)$ & 0.13 \\
\hline Shallow well & $1(3)$ & $3(4)$ & $0.6(0.0-13.4)$ & 1.00 \\
\hline Household member with cholera & $13(37)$ & $19(26)$ & $1.6(0.6-4.4)$ & 0.37 \\
\hline \multicolumn{5}{|l|}{ Type of toilet } \\
\hline Pit latrine & $36(95)$ & $59(81)$ & $4.2(0.9-40.4)$ & 0.08 \\
\hline Flush toilet & $2(5)$ & 14 (19) & Ref & - \\
\hline \multicolumn{5}{|l|}{ Household shares latrine } \\
\hline Yes & $30(83)$ & $60(80)$ & $1.3(0.5-4.4)$ & 0.76 \\
\hline No & $6(17)$ & $15(20)$ & Ref & \\
\hline \multicolumn{5}{|l|}{ Cholera vaccination\# } \\
\hline Yes & $5(13)$ & $10(14)$ & $0.9(0.2-3.4)$ & 1.00 \\
\hline No & $33(87)$ & $64(86)$ & Ref & - \\
\hline
\end{tabular}

* Numbers do not always sum to total because of missing values.

$\dagger$ Age analysis was conducted only on age $\geq 18$ years, as age was not matched in that age-group, and the only matching variable for that analysis was date of onset. $\ddagger$ Only adults (aged $\geq 18$ years) were considered for analysis of education level.

$\S$ Employment status only analyzed for those aged $16-65$ years.

\| Multiple-answer choices allowed.

I Shallow well, kiosk, borehole, and piped water were the only selected responses among other options. The mOR compares each category to the other three categories.

\# At least one dose.

reported leg cramps, fever, and headache (Table 3). The travel time to a health facility was not statistically different among decedents (median: 25 minutes [interquartile range $\{\mathrm{IQR}\}$ 20-38]) and survivors (median: 30 minutes [IQR 15-30], $P=$ 0.85). The time from illness onset to arrival at a health care facility (for those who received clinical care) was similar among decedents and survivors, with nearly half of the patients $(44 \%$ and $43 \%$, respectively) arriving more than 6 hours after illness onset. Among those presenting to a healthcare facility for treatment, decedents had decreased odds of immediately receiving oral rehydration solution (ORS) (mOR $0.1,95 \% \mathrm{Cl}$ : $0.0-0.6, P=0.02$ ) and intravenous fluids (IVF) (mOR 0.3, 95\%

TABLE 2

Care at home before presentation at a cholera treatment center among cholera patients, by survivorship status-Zambia, 2017-2018

\begin{tabular}{|c|c|c|c|c|}
\hline & Decedents $(n=38), n^{\star}(\%)$ & Survivors $(n=76), n^{\star}(\%)$ & Matched odds ratio $(95 \% \mathrm{Cl})$ & $P$-value \\
\hline \multicolumn{5}{|l|}{ Received ORS } \\
\hline Yes & $14(40)$ & $27(36)$ & $1.2(0.5-3.0)$ & 0.82 \\
\hline No & $21(60)$ & $48(64)$ & Ref & - \\
\hline $\begin{array}{l}\text { Time (hours) from illness onset to initiation } \\
\text { of ORS, median [IQR] }\end{array}$ & $2[1-7]$ & $2[1-5]$ & - & $0.82 \dagger$ \\
\hline \multicolumn{5}{|l|}{ Received antibiotics } \\
\hline Yes & $8(21)$ & $13(17)$ & $1.3(0.4-3.7)$ & 0.79 \\
\hline No & $30(79)$ & $62(83)$ & Ref & - \\
\hline
\end{tabular}

* Numbers do not add up to total for some variables because of missing values.

† Wilcoxon rank-sum test. 
TABLE 3

Clinical symptoms during the week of illness and clinical care of cholera patients, by survivorship status-Zambia, 2017-2018*

\begin{tabular}{|c|c|c|c|c|}
\hline & Decedents $(n=21), n \dagger(\%)$ & Survivors $(n=42), n \dagger(\%)$ & Matched odds ratio $(95 \% \mathrm{Cl})$ & $P$-value \\
\hline \multicolumn{5}{|l|}{ Clinical symptoms } \\
\hline \multicolumn{5}{|l|}{ Diarrhea } \\
\hline Yes & $16(80)$ & $41(98)$ & $0.1(0.0-1.3)$ & 0.09 \\
\hline No & $4(20)$ & $1(2)$ & Ref & - \\
\hline \multicolumn{5}{|l|}{ Vomiting } \\
\hline Yes & $12(57)$ & $33(79)$ & $0.4(0.1-1.4)$ & 0.16 \\
\hline No & $9(43)$ & $9(21)$ & Ref & - \\
\hline \multicolumn{5}{|l|}{ Leg cramps } \\
\hline Yes & $6(40)$ & $12(29)$ & $1.4(0.2-8.2)$ & 0.88 \\
\hline No & $9(60)$ & $29(71)$ & Ref & - \\
\hline \multicolumn{5}{|l|}{ Fever } \\
\hline Yes & $5(31)$ & $5(11)$ & $7.2(0.7-355)$ & 0.11 \\
\hline No & $11(69)$ & $36(88)$ & Ref & - \\
\hline \multicolumn{5}{|l|}{ Headache } \\
\hline Yes & $2(14)$ & $5(13)$ & $1.3(0.1-11.6)$ & 1.00 \\
\hline No & $12(86)$ & $35(87)$ & Ref & - \\
\hline \multicolumn{5}{|l|}{ Clinical care } \\
\hline \multicolumn{5}{|c|}{ Received oral rehydration solution } \\
\hline Immediately & $2(29)$ & $24(73)$ & $0.1(0.0-0.6)$ & 0.02 \\
\hline Not immediately & $5(71)$ & $9(27)$ & Ref & - \\
\hline \multicolumn{5}{|c|}{ Received intravenous fluids } \\
\hline Immediately & $3(38)$ & $26(74)$ & $0.3(0.0-2.0)$ & 0.29 \\
\hline Not immediately & $5(62)$ & $9(26)$ & Ref & - \\
\hline \multicolumn{5}{|l|}{ Received antibiotics } \\
\hline Yes & $6(67)$ & $24(73)$ & $1.5(0.1-79)$ & 1.00 \\
\hline No & $3(33)$ & $9(27)$ & Ref & - \\
\hline \multicolumn{5}{|c|}{$\begin{array}{l}\text { Time from illness onset to arrival } \\
\text { at CTC (hours) }\end{array}$} \\
\hline$\leq 6$ & $9(56)$ & $28(57)$ & Ref & 1.00 \\
\hline$>6$ & $7(44)$ & $21(43)$ & $0.8(0.2-3.5)$ & - \\
\hline \multicolumn{5}{|c|}{ Duration of stay in CTC (hours) } \\
\hline$\leq 12$ & $6(35)$ & $11(26)$ & Ref & - \\
\hline$>12$ & $11(65)$ & 31 (74) & $0.6(0.1-3.1)$ & 0.71 \\
\hline
\end{tabular}

CTC $=$ cholera treatment center.
${ }^{*}$ Excludes community decedents and their matched survivors.

† Numbers do not add up to total for some variables because of missing values.

Cl: $0.0-0.2, P=0.29)$, although the latter was not statistically significant. Most respondents reported that decedents and survivors received antibiotics in the CTC $(67 \%$ and $73 \%$, respectively) and stayed in the CTC longer than 12 hours $(65 \%$ and $74 \%$, respectively).

Location. Seven CTCs were in operation during the cholera outbreak in Lusaka district. The mean distance traveled between the patient's home and the nearest CTC was $2.2 \mathrm{~km}$ $(\mathrm{SD} \pm 1.7 \mathrm{~km})$, and no statistical difference was found between the distances that decedents and survivors traveled $(2.3 \mathrm{~km}$ versus $2.2 \mathrm{~km}$, respectively, $P=0.49$ ).

Knowledge and practices. Most respondents had heard of cholera, knew that cholera could be prevented and treated, and knew that a person should go to a hospital immediately upon getting sick (Table 4). Most respondents (83\%) reported treating their drinking water on the day of the interview; approximately half reported they had treated their drinking water before illness. Responses for decedents and survivors were not statistically different (all $P>0.05$ ).

Chart reviews. Apart from the case-control study, a detailed review of available decedent charts was completed; some, but not all, of these charts represented patients also included in the case-control study. Of 24 decedents' charts provided for review from four CTCs, 10 (42\%) described patients who had died in the community or died within 45 minutes of arrival and were excluded from the following analyses. Of the 14 remaining, eight (57\%) were female, and the median age was 37.5 years (range $2-88$ years). The median length of stay was 27 hours $(n=12)$. Three $(21 \%)$ were documented as HIV positive, whereas the status of the remainder was undocumented. Four (57\%) of seven patients with documentation had dehydration described as severe on admission. All but one patient (93\%) received IVF, but because of limited documentation, we were unable to determine if IVF treatment matched national treatment protocols. Ten $(71 \%)$ received an antibiotic recommended for cholera treatment. Twelve (92\%) of 13 with documentation were positive for cholera by rapid diagnostic testing (RDT). Of the 24 charts reviewed, none had documentation of stool culture results to confirm cholera infection.

\section{DISCUSSION}

During the early weeks of the cholera outbreak in December 2017 , cholera mortality approached $3 \%$, with one-quarter of deaths occurring in the community. The risk factors for cholera mortality identified in this study were as follows: age $>55$ years and not having completed primary school. Receiving ORS immediately on presentation to a CTC was protective. The relatively small sample size of our study may have limited our ability to detect other factors previously associated with cholera mortality in other places and settings.

Early access to rehydration (such as ORS and IVF) is the cornerstone of clinical management for cholera. The use of 
TABLE 4

Knowledge and behaviors of cholera and drinking water treatment, by survivorship status_Lusaka, Zambia, 2017-2018 Decedents $(n=38), n^{*}(\%)$ Survivors $(n=76), n^{\star}(\%)$ $\mathrm{mOR}(95 \% \mathrm{Cl})$

\begin{tabular}{|c|c|c|c|c|}
\hline \multicolumn{5}{|l|}{ Knowledge } \\
\hline \multicolumn{5}{|c|}{ Heard of cholera } \\
\hline Yes & $31(89)$ & $67(92)$ & $0.7(0.1-4.4)$ & 0.99 \\
\hline No & $4(11)$ & $6(8)$ & Ref & - \\
\hline \multicolumn{5}{|c|}{ Cholera can be prevented } \\
\hline Yes & $32(97)$ & $67(96)$ & $1.0(0.1-59.0)$ & 1.00 \\
\hline No & $1(3)$ & $3(4)$ & Ref & - \\
\hline \multicolumn{5}{|c|}{ Cholera can be treated } \\
\hline Yes & $33(100)$ & $67(96)$ & 1.9 (0.3-infinity) & 0.59 \\
\hline No & $0(0)$ & $3(4)$ & Ref & - \\
\hline \multicolumn{5}{|c|}{$\begin{array}{l}\text { Should go to hospital } \\
\text { immediately }\end{array}$} \\
\hline Yes & $35(92)$ & $72(95)$ & $0.7(0.1-4.6)$ & 0.86 \\
\hline No & $3(8)$ & $4(5)$ & Ref & - \\
\hline \multicolumn{5}{|l|}{ Behaviors } \\
\hline \multicolumn{5}{|c|}{$\begin{array}{l}\text { Treated drinking water } \\
\text { before illness2 }\end{array}$} \\
\hline Yes & $22(63)$ & $35(47)$ & $1.8(0.7-4.7)$ & 0.23 \\
\hline No & $13(38)$ & $40(53)$ & Ref & \\
\hline \multicolumn{5}{|c|}{$\begin{array}{l}\text { Treated drinking water } \\
\text { today } \dagger\end{array}$} \\
\hline Yes & $30(79)$ & $63(85)$ & $0.6(0.1,2.1)$ & 0.47 \\
\hline No & $8(21)$ & $11(15)$ & Ref & - \\
\hline
\end{tabular}

† Drinking water teatment methods repoted were chlorination and/or bolling.

ORS has been associated with decreased mortality from diarrhea in infants and children aged $<5$ years, ${ }^{10,11}$ and increasing the availability of ORS in the community has been shown to decrease mortality from cholera. ${ }^{12-15}$ This study supports these previous findings, as receiving ORS immediately at the healthcare facility was identified as a protective factor. Unlike previous studies, ${ }^{12,13}$ this study did not demonstrate an association between receiving ORS at home and mortality. The short distance necessary to travel to a CTC (mean: $2 \mathrm{~km}$ ) in this peri-urban outbreak may explain why home ORS treatment was not shown to provide the protection noted in previous studies. ${ }^{12,13}$ Home ORS may be most critical to bridge the gap when patients travel longer distances to receive additional health care.

Infection with cholera is more common in communities with a lower socioeconomic status (SES), likely due in part to limited access to safe water and sanitation. However, there are limited published data related to SES and cholera mortality among those infected. A previous study in Cameroon has highlighted an association between lower SES and cholera mortality. ${ }^{12}$ Our study did not find a statistically significant association between proxies for lower SES (such as use of pit latrines and household assets) and cholera mortality. In a previous study, household ownership of a mobile phone, which may represent a higher relative SES, was found to be associated with decreased cholera severity. ${ }^{16}$ Although our data did not demonstrate a statistically significant association between smart phone ownership and decreased cholera mortality, mobile phone ownership may improve a patient's ability to communicate and seek timely health care, which merits further exploration. Lower SES has also been associated with fewer years of education. ${ }^{17}$ However, this is the first study to identify lower education as a risk factor for cholera mortality. ${ }^{12,13,18-22}$ The increased odds of cholera mortality in those with lower education levels, in the context of an affected population with potentially similar SES, emphasizes the importance of education as a key contributor to one's health and well-being. A lower education level can inhibit one's ability to protect oneself from diseases, such as cholera. Limited literacy can also affect a person's ability to receive health messaging, which may impact survival.

In our study, a higher odds of mortality was found among adults with increased age ( $>55$ years), which was similar to findings from previous studies in Cameroon, Haiti, and Guinea Bissau. ${ }^{12,19,21,22}$ Previous studies have shown mixed results regarding the relationship between cholera mortality and gender. Although several studies have identified male gender as a risk factor for mortality, ${ }^{13,19}$ no association was seen in this study, which is consistent with other published reports. ${ }^{12,20-22}$

Appropriate clinical management is essential for decreasing cholera mortality. Severe cholera leads to rapid dehydration due to large volumes of stool output (as high as one liter per hour, in adults), which requires close monitoring and aggressive rehydration with ORS and IVF. With proper rehydration, the CFR for cholera should be less than $1 \% .^{6,7}$ In the chart review, we were unable to assess the adequacy of rehydration due to limited documentation. However, the reported duration between admission and death for most decedents who received care at a CTC was greater than 12 hours, which should be adequate time for appropriate fluid resuscitation in patients without complications. ${ }^{20}$ The case-control investigation and the chart review demonstrated frequent use of antibiotics, which are recommended for severe cholera and selected highrisk patients. Unlike a previous study, ${ }^{20}$ neither home nor hospital-administered antibiotics demonstrated statistically significant protection from death in this setting.

The chart review also raised concerns regarding the appropriate use of RDT and the impact RDTs may have on clinical management. Three of 13 patients with documentation initially tested negative by RDT. One of these patients was 
then transferred out of the CTC, despite meeting the cholera case definition, to the female ward where she died a few hours later. The other two patients initially testing negative were retested and found to be positive by RDT after death (or on documentation certifying death). Because of limited documentation, it is unclear if inaccurate RDT results altered clinical decision-making. Current recommendations by the Global Task Force on Cholera Control advise that RDTs are intended for surveillance purposes in peripheral healthcare facilities and have limited utility for individual diagnosis as the results would have no influence on the immediate management of the patient with suspected cholera, which should be guided by clinical assessment of dehydration and fluid losses and on cholera treatment guidelines. ${ }^{23}$ Rapid diagnostic test results are not reliable enough to influence individual treatment decisions of suspected cholera cases during a cholera outbreak.

The increasing availability of oral cholera vaccines (OCVs) has provided an additional tool for cholera control and prevention. A single-dose OCV campaign targeting $>500,000$ people was conducted in Lusaka in April 2016. A second dose was offered to persons at risk in December $2016 .{ }^{24,25}$ The average two-dose effectiveness of the killed OCV has been demonstrated to be $76 \%$ at 18 months (weighted mean duration) in a systematic review. ${ }^{26}$ Similarly, in Haiti, two-dose effectiveness has been reported as $76 \%$ at 4 years. ${ }^{27}$ Decreased efficacy has been documented in patients aged less than 5 years and beyond the second year after administration. ${ }^{26-28}$ With this knowledge, we asked patients and their proxies if the patient received zero, one, or two doses of the OCV. According to respondents, only four (4\%) patients had previously received two doses of OCV, and a minority of decedents (13\%) and survivors (14\%) had previously received one or more doses of OCV. No statistically significant difference was noted. However, these results should be interpreted cautiously as vaccination was not validated by vaccination records. Recall bias and responses from proxies may have led to inaccuracies in reporting. Future studies should consider investigating the relationship between OCV and cholera mortality more comprehensively.

This investigation has several limitations. First, in the casecontrol study, we attempted to include all cholera deaths occurring during the study time period and to select a representative sample of matched controls. However, we encountered challenges in locating the selected decedents and survivors because of a number of factors. For example, some patients (or patients' families) provided incorrect information at the CTC, including false names and incorrect or incomplete addresses. We received reports that some had voluntarily relocated to another community (or had been forced to move by a landlord) because of stigma from the patient's illness with cholera. In addition, there were errors in the line list, including patient ages, names, addresses, and outcomes, that made it difficult to trace each patient. We collaborated with community health workers to assist our efforts, but we were unable to locate all the selected decedents and survivors. A second limitation is that although the questionnaires were administered within 4 months of the patient's illness (median: 30 days; range 5-99 days), recall bias by patients or their proxies may have been introduced, given the time duration between illness and interview. Another limitation, response bias, is introduced when interviewing proxy respondents. To attenuate this risk, we attempted to interview proxies that were primary caregivers and those who provided support during the illness. However, as many studies have described, ${ }^{29}$ proxy respondents may introduce biases and inaccuracies, especially as the relationship distance widens. For example, we suspect that inaccurate recollections and responses from proxies may have contributed to underreporting of diarrhea in decedents. Fourth, because this study was limited to a relatively small sample size of 38 decedents and 76 survivors (or less), we could not further examine the joint contribution of factors to cholera mortality in a multivariable analysis. Last, there is a risk of misclassification of illness. Not all participants had confirmatory testing for cholera by stool culture. Although all study participants reportedly met the case definition for suspected cholera as evidenced by their inclusion on the line list, some patients may have experienced an illness unrelated to cholera.

Our study highlights the increased risk for cholera mortality in adults aged $>55$ years and among patients with lower education levels. It also underscores the importance of early rehydration with ORS. These findings can help guide messaging during future outbreaks, emphasizing the need to reach all community members, including those with advanced age and limited education. Communication strategies used during an outbreak response should consider the literacy level (including health literacy) of those most at risk for death. Messages should be simple and should include spoken messages in the primary language of those at risk. Messages should also be shared via platforms accessible to and used by older adults. Traditional cholera prevention and treatment messages remain relevant to prevent deaths during outbreaks. These include underscoring the importance of drinking ORS at home for mild illness and that all suspected cholera patients should seek health care immediately if symptoms worsen or dehydration occurs. Because rehydration remains the cornerstone of cholera treatment, public health interventions should include increasing availability of ORS in the community and facilitating ease of access to treatment at healthcare facilities with a focus on early rehydration with ORS and IVF to decrease cholera mortality.

Received September 11, 2019. Accepted for publication November 16, 2019.

Published online January 13, 2020.

Acknowledgments: We would like to acknowledge the contributions of José Aponte, Orbrie Chewe, Chanda Chikwanda, Moses Chilufya, Chimwemwe Waya, William Davis, Raymond Hamoonga, Yahya Kandeh, Nathan Kapata, Muzala Kapina, Shadreck Kayeye, Chileshe Mabula, Warren Malambo, Clevina Mizanda, Albertina Moraes, Fortunate Mutesi, Francis D. Mwansa, Chikoloma Nakazwe, Rupa Narra, Ezinne Onwuekwe, Margo Riggs, Namundi Siwale, and Ann Williams.

Disclosure: The findings and conclusions in this report are those of the authors and do not necessarily represent the official position of the Centers for Disease Control and Prevention.

Authors' addresses: Lwito Salifya Mutale, Patrick Sakubita, Fred Kapaya, Nelia L. Mulambya, Francis H. Nanzaluka, Angela Gama, and Vivian Mwale, Zambia Field Epidemiology Training Program, Lusaka, Zambia, E-mails: Iwitosalifyanji@gmail.com, sakubitap@gmail.com, fkapaya2007@gmail.com, nmulambya2000@gmail.com, nanzilf@ yahoo.com, angelagama13@yahoo.com, and vivuva@gmail.com. Alison Winstead, Sunkyung Kim, Eric Mintz, and Joan Brunkard, Centers for Disease Control and Prevention, Atlanta, GA, E-mails: nrc0@cdc.gov, wox0@cdc.gov, edm1@cdc.gov, and feu4@cdc.gov. Sulani Nyimbili, Zambian Ministry of Health, Lusaka, Zambia, E-mail: sulanins@gmail.com. William Ngosa, Nyambe Sinyange, and Victor Mukonka, Zambia National Public Health Institute, Lusaka, Zambia, E-mails: ngosawilliam@gmail.com, bsinyange@gmail.com, and vmukonka@gmail.com. Ellen Yard, Centers for Disease Control and Prevention, Lusaka, Zambia, E-mail: igf8@cdc.gov. 


\section{REFERENCES}

1. Ali M, Nelson AR, Lopez AL, Sack DA, 2015. Updated global burden of cholera in endemic countries. PLoS Negl Trop Dis 9: e0003832.

2. World Health Organization, Global Task Force on Cholera Control, 2011. Cholera Country Profile: Zambia. Available at: www.who.int/ cholera/countries/ZambiaCountryProfile2011.pdf. Accessed August 31, 2018.

3. Mazaba ML, 2018. 2017/2018 Cholera Outbreak Response Media Briefing - The Health Press - Zambia. Available at: http:// znphi.co.zm/thehealthpress/2017-2018-cholera-outbreakresponse-media-briefing/. Accessed January 7, 2019.

4. Olu O, Babaniyi O, Songolo P, Matapo B, Chizema E, Kapin'aKanyanga M, Musenga E, Walker O, 2013. Cholera epidemiology in Zambia from 2000 to 2010: implications for improving cholera prevention and control strategies in the country. East Afr Med J 90: 324-331.

5. Sasaki S, Suzuki H, Igarashi K, Tambatamba B, Mulenga P, 2008. Spatial analysis of risk factor of cholera outbreak for 2003-2004 in a peri-urban area of Lusaka, Zambia. Am J Trop Med Hyg 79: 414-421.

6. United Nations Children's Fund, 2017. UNICEF Cholera Toolkit 2013. Available at: https://www.unicef.org/cholera/index 71222.html. Accessed May 23, 2019.

7. Davis W, Narra R, Mintz ED, 2018. Cholera. Curr Epidemiol Rep 5: 303.

8. Central Statistical Office, 2014. 2010 Census of Population and Housing - Lusaka Province Analytical Report. Available at: https://www.zamstats.gov.zm/index.php/publications/category/ 58-2010-census-analytical-reports. Accessed May 13, 2019.

9. WHO/UNICEF, 2017. Joint Monitoring Programme Global Database. Available at: https://washdata.org/data. Accessed May 13, 2019

10. Victora CG, Bryce J, Fontaine O, Monasch R, 2000. Reducing deaths from diarrhoea through oral rehydration therapy. Bull World Health Organ 78: 1246-1255.

11. Boschi-Pinto C, Bahl R, Martines J, 2009. Limited progress in increasing coverage of neonatal and child-health interventions in Africa and Asia. J Health Popul Nutr 27: 755-762.

12. Cartwright EJ, Patel MK, Mbopi-Keou FX, Ayers T, Haenke B, Wagenaar BH, Mintz E, Quick R, 2013. Recurrent epidemic cholera with high mortality in Cameroon: persistent challenges 40 years into the seventh pandemic. Epidemiol Infect 141: 2083-2093.

13. Morof $D$ et al., 2013. Community mortality from cholera: urban and rural districts in Zimbabwe. Am J Trop Med Hyg 88: 645-650.

14. Sircar BK, Saha MR, Deb BC, Singh PK, Pal SC, 1990. Effectiveness of oral rehydration salt solution (ORS) in reduction of death during cholera epidemic. Indian J Public Health 34: 68-70.
15. Mahalanabis D, Choudhuri AB, Bagchi NG, Bhattacharya AK, Simpson TW, 1973. Oral fluid therapy of cholera among Bangladesh refugees. Johns Hopkins Med J 132: 197-205.

16. Jackson BR et al., 2013. Seroepidemiologic survey of epidemic cholera in Haiti to assess spectrum of illness and risk factors for severe disease. Am J Trop Med Hyg 89: 654-664.

17. Poverty Practice in the Bureau for Development Policy, United Nations Development Programme, 2013. Humanity Divided: Confronting Inequality in Developing Countries. Available at: https://www.undp.org/content/undp/en/home/librarypage/povertyreduction/humanity-divided--confronting-inequality-in-developingcountries.html. Accessed May 23, 2019.

18. McCrickard LS et al., 2017. Cholera mortality during urban epidemic, dar es Salaam, Tanzania, August 16, 2015-January 16, 2016. Emerg Infect Dis 23 (Suppl): S154-S157.

19. Gunnlaugsson G, Angulo FJ, Einarsdóttir J, Passa A, Tauxe RV, 2000. Epidemic cholera in Guinea-Bissau: the challenge of preventing deaths in rural west Africa. Int $J$ Infect Dis 4: 8-13.

20. Shikanga OT, Mutonga D, Abade M, Amwayi S, Ope M, Limo H, Mintz E, Quick R, Breiman R, Feikin D, 2009. High mortality in a cholera outbreak in western Kenya after post-election violence in 2008. Am J Trop Med Hyg 81: 1085-1090.

21. Djouma FN, Ateudjieu J, Ram M, Debes AK, Sack DA, 2016. Factors associated with fatal outcomes following cholera-like syndrome in far north region of Cameroon: a community-based survey. Am J Trop Med Hyg 95: 1287-1291.

22. Page A-L, Ciglenecki I, Jasmin ER, Desvignes L, Grandesso F, Polonsky J, Nicholas S, Alberti K, Porten K, Luquero F, 2015. Geographic distribution and mortality risk factors during the cholera outbreak in a rural region of Haiti, 2010-2011. PLOS Negl Trop Dis 9: e0003605.

23. Global Task Force on Cholera Control Surveillance Working Group, 2016. The Use of Rapid Diagnostic Tests. Available at: https://www.who.int/cholera/task_force/Interim-guidancecholera-RDT.pdf?ua $=1$.

24. Poncin $\mathrm{M}$ et al., 2017. Implementation research: reactive mass vaccination with single-dose oral cholera vaccine, Zambia. Bull World Health Organ 96: 86-93.

25. Ferreras $E$ et al., 2018. Single-dose cholera vaccine in response to an outbreak in Zambia. N Engl J Med 378: 577-579.

26. Bi Q et al., 2017. Protection against cholera from killed whole-cell oral cholera vaccines: a systematic review and meta-analysis. Lancet Infect Dis 17: 1080-1088.

27. Franke MF, Ternier R, Jerome JG, Matias WR, Harris JB, Ivers LC, 2018. Long-term effectiveness of one and two doses of a killed, bivalent, whole-cell oral cholera vaccine in Haiti: an extended case-control study. Lancet Glob Health 6: e1028-e1035.

28. Sur $D$ et al., 2011. Efficacy of a low-cost, inactivated whole-cell oral cholera vaccine: results from 3 years of follow-up of a randomized, controlled trial. PLoS Negl Trop Dis 5: e1289.

29. Elliott MN, Beckett MK, Chong K, Hambarsoomians K, Hays RD, 2008. How do proxy responses and proxy-assisted responses differ from what medicare beneficiaries might have reported about their health care? Health Serv Res 43: 833-848. 